\title{
A Study on the Effects and Significance of Liu Miri's Family Novels
}

\author{
Aiping Fang \\ School of Foreign Studies, Yangtze University, Jingzhou City, Hubei \\ Province, 434000, China
}

\begin{abstract}
Liu Miri is a famous writer in Japanese contemporary literature. Her unique family novels not only have the profound social contents and practical significance, but also have unique styles and distinctive characters, which add a unique landscape to the development of modern Japanese Literature. Based on the author's learning and practical experience, this paper first analyzed Liu Miri's creation experience, and then discussed the causes of the theme of "family" in her works, and finally discussed the significance and influence of Liu Miri's family novels.
\end{abstract}

Key words: Liu Miri; Family Novel; Novel; Meaning; Influence

\section{Introduction}

Liu Miri is a famous contemporary Japanese writer, born in 1968 in Yokohama, Kanagawa, Japan, and she is the third generation of South Koreans emigrated to Japan. Since she does not change her nationality, strictly speaking, Liu Miri is a Korean in Japan rather than a Korea Japanese[1]. Although Liu Miri once said that she is neither Japanese nor Korean, just creates novels to skip over the gap between herself and others, but because of her creation depends on Japanese contemporary social environment and cultural atmosphere, plus that she writes with standard Japanese, and her novels have a unique representation in contemporary female literature in Japan, so her novels have important influence on contemporary Japanese literature. 


\section{Liu Miri's Creation Experience}

As we all know, Japan is a nation with great cultural cohesion, but it also has a strong exclusiveness. The background for foreigners living in Japan will result in searching for themselves in the narrow crevice between the state and national consciousness, thus it must be very hard. However, although the writer Liu Miri grew in such a crevice, she does not stop writing. Due to the identity of the Korean in Japan, Liu Miri had been discriminated and bullied by teachers and students and became the object of tease during the reading period, so her eccentric and excess self awareness personality was influenced by the experience of childhood. Juvenile life caused great harm to Liu Miri but it also became the source of creation in her adult life[2]. Liu Miri restored her life with sharp strokes, and tried to find their true balance and psychological comfort in the creation.

In addition to drama and fiction, Liu Miri also tried writing essays. So far, the published works are: "Liu Miri's Suicide" (1995), which discusses the suicide problem; "Family Specimen" (1995), which shows contemporary Japanese family sample, comments set "From a Window Bookstore" (1996), "Whisper Dictionary" (1996), which uniquely defines different kinds of things; autobiographical essays "Cradle by the Edge of the Water" (1997); "Language lesson" (1998), which highlights the various social problems. Some of these works, such as "family sample", is more like what we call "micro novel", each chapter of these novels can deeply affect readers' perception of different types of families.

\section{Reasons for the Theme of "Family" in Liu's Works}

The form of Japan's long feudal society is patriarchy, thus women's rights of discourse had not been fully recognized and guaranteed by the society. After World War II, with the development of society, the increase of income and the improve of the personal ability of Japanese women, women started to require the full realization of the corresponding social status and the right to speak. Thus, the traditional patriarchal family model had deviated from the concept of modern equality and democratic society, and it especially did not meet the requirements of the new time women, then, the Japanese contemporary writers, represented by Liu Miri, first broke this tradition and explored the new family in literary world. From the challenge to traditional family values to the collapse of family system, then to the establishment of the new concept of family after the disintegration of family system, from using the female perspective to understand themselves and determining their nature and values to looking at the outside world, and to grasp and understand the feminine life characteristics[3]. The Japanese contemporary female writers, represented by Liu Miri, always use their keen consciousness to correct the one-way visual angle and the right of speech from the perspective of men. Objects they focused on widely extended from the opposite relationship 
between men and women to such as the relationship between the spirit and the body, reality and fantasy.

In a word, Liu Miri writes the exploration and pursuit of a new type of family in her works, which to a certain extent can cause thinking of the community, she is not to completely change the social pattern, but let people reflect on the disadvantages and problems of traditional family in the wonderful exploration, in order to make the family model better adapts to the development of today's society. After all, the family is the basic unit of society, only harmonious family can create a harmonious society, thus the step of human progress will never cease.

\section{The Influence and Significance of the Liu Miri's Family Novels}

\subsection{The influence of the Liu Miri's family novels}

With the awakening of female self-consciousness, natural relationship between women and family decided that the creation of female writers first took the family as the starting point. While paying attention to women's survival chances and fates, the Japanese contemporary female writers, such as Liu Miri, issued a challenge to the traditional family views. Although different female writers have different styles, they all revolted the established tradition of male chauvinism and promoted the strong sense of life and the consciousness in their own personalities. In Liu Miri's exploration process, the need to build the family became her ultimate attribution, and this new family model which beyond the family blood and marriage ties expressed the author's desire to reproduce the warmth of the family. In addition, the consciousness of Liu Miri has been resonated with a lot of young women writers, and has been promoted within a certain range[4].

Further analyzing contemporary Japanese writer's works, it is not difficult to find that they do not only highlight the expression of female experience and the display of female desire, but also own a deep examination of tradition, deconstruction of patriarchal culture and unique understanding of art. The meaning of deconstruction lies in the reconstruction, what they want is to establish a art world which is independent and is full of gender characteristics but beyonds gender confrontation, and this construction no longer belongs to sex rights, but is multi angle and multi-level. "Cradle by the edge of the water" can be said to be the rebellion against Liu Miri's thought of contemporary traditional ideas. Although there are many modern works reveal the internal contradictions of the broken families, Liu Miri can use dispassionate tone to expose her heart scar. Her novels are neither humble nor pushy and do not detain the dead family, but bring people to the process of the disintegration of the family and thus reflect the social significance. Japanese literary critic Kawamura Nato once said: "with the progress of society and more and more women going to the community, the disintegration process of the family in Japanese society will be faster and faster, and the degree will deeper. Female writers, represented by Liu Miri, are not only the participants of the profound changes, but also the narrator of the process. Liu 
Miri can face the family misfortune calmly and honestly, and her plain depiction makes people think deeply.

\subsection{Influence of Liu Miri's Family Novels}

(1) Influence of Liu Miri's novels on Japanese society

Liu's works, which theme is "family collapse" reflect the changes of the family and the relationship between the sexes in the contemporary urban life in Japan, and women's disintegration and renewal of traditional ideas on these issues. These works, to a certain extent, criticize some puzzling problems in the current Japanese society, thus we can see the bold expression of the feminist consciousness and the new women's consciousness. Liu Miri more naturally writes the passions and women's own love and hate on the basis of personal experience and personal feelings, reveals the anxious, depressed, lonely moods of modern people in different cultural impacts according to her own experience.

Japanese literary critic Fukuda Kazuno also supports Liu Miri in this matter. $\mathrm{He}$ believes that there is no absolute freedom of expression in the world, also there is no absolute respect for human rights, that reality is not exist, and the literary world can not be completely corresponding to the real world, so there is no absolute justice in literature, otherwise the writer cannot write novels. Only in the relative world, we can distinguish the true from the false and distinguish the good from evil in the literature[5]. Liu Mei Li believes that literature is unable to cut off the contact with reality, and in Japan, the writing tradition of "private novel" is also persistent. The so-called "private novel", an accepted explanation is from Takahashi Hideo: using the character who is almost equal to the author as the protagonist, describing the person's daily life, or describing the character's love and moods. A good private novel should be a pair of selfishness and selflessness. If Liu's works infringe the privacy of others, then the past private novels will have the suspicion of violating human rights.

(2) Influence of Liu Miri's novels on the development of women's literature in the world

Since 1990s, in the relatively slow development period of the Western feminist literature, Japanese female writers, represented by Liu Miri, supported the development of the world's feminist literature through their works, and they formed a good supporting role in practice and formed the trend of mutual consideration with Western women writers and feminists. Woolf, a British female writer, has a clear sense of the uniqueness of female writing. She believes that the reason why women write different contents compared to men is not because they are psychologically different, but because they are different from men in social status. Therefore, her intention of writing women's experience is to find out the language form which is limited to the female life, and she believes that when women finally win the equality with men in social and economic status, there is no force can stop them fully revealing their artistic talents.

The long - term practice of Japanese women writers and the western feminist movement all resist the traditional relationship between the sexes. Under the significant suppression of the patriarchal culture, women succumb to men in 
culture, history, society and gender relations. Although there are many different expressions of feminist criticism, they are still struggling to break from the natural concept of patriarchal literature and criticism. It means that it refuses to be incorporated into any particular method and tries to disrupt and subvert all established theoretical practices.

Liu's novels are mostly based on her family, and readers tend to classify them as "private novels", while Mari Liu had denied this. Maybe the material comes from the family, but not all the stories are true. Such as the homeless in the "home dream is far away" is a fictional plot. So Liu Miri's novels indirectly used the creation mode of "private novel" to explore a new female literary creation, this exploration was not only the ornament to the Japanese female literature, but also been known by the world because of winning the Akutagawa prize for literature, and it injected fresh blood and energy for the further development of the world female literature.

\section{Conclusion}

Although the domestic research results of contemporary Japanese women's literature are rich and colorful, there are not many articles discuss the collection of works of a Japanese female writer. Through the analysis of works, this paper expounded the theme, the characters and the social significance and the influence of the contemporary Japanese female literature representative writer Liu's family novels. This paper argued that the establishment of a new family is very important in the eyes of contemporary Japanese women writers, and proved that the works and ideas of the new generation of Japanese women writers, represented by Liu Miri, have an important influence on the development of women's literature in the world.

\section{References}

[1] Li Jianyun. Liu Miri and Her Works. Foreign Literature, 1999, 05:8-11.

[2],Zhang Xiaoning, Bao Shulan.. The Family Theme of Liu Miri's Literary Works. Japanese Studies, 2012, 01:77-81.

[3] Zhang Wenying, Jiang Haiyan. Watch the Disintegration of the Contemporary Family from Liu Miri's "family film". The study and research of Japanese, 2009, 04:93-98.,

[4] Chuan Cun Ji, Xu Jinlong. Japanese Modern Women and "Family". World Literature, 2001, 04:254-263.

[5] Fu Xia heron. The Family Values of Japanese Contemporary Female Literature. Century Bridge, 2007, 10:85+91. 